\title{
Changes in serum IGF-1 level and tumor VEGF expression in mice with colorectal cancer under hyperglycemic conditions
}

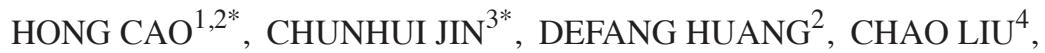 \\ DAOQI SUN ${ }^{2}, \mathrm{CHENG} \mathrm{TAN}^{5}$, XIAOMING ZHU ${ }^{3}$ and YAJUN FEI ${ }^{3}$ \\ ${ }^{1}$ Department of Endocrinology, Nanjing University of Chinese Medicine, Nanjing, Jiangsu 210046; \\ ${ }^{2}$ Department of Endocrinology, The Third Affiliated Hospital of Nantong University; ${ }^{3}$ Department of Oncology, \\ Wuxi Traditional Chinese Medicine Hospital, Wuxi, Jiangsu 214001; ${ }^{4}$ Department of Endocrinology, \\ Jiangsu Province Hospital on Integration of Chinese and Western Medicine, Nanjing, Jiangsu 210028; \\ ${ }^{5}$ Jiangsu Institute of Nuclear Medicine, Wuxi, Jiangsu 214063, P.R. China
}

Received December 22, 2012; Accepted February 18, 2013

DOI: $10.3892 / \mathrm{mmr} .2013 .1339$

\begin{abstract}
The present study aimed to observe the growth of transplanted tumors in mice with colorectal cancer (CRC) under hyperglycemic conditions and to detect the expression of vascular endothelial growth factor (VEGF) in these tumors. The study also aimed to observe the changes in serum insulin-like growth factor-1 (IGF-1) levels and to determine whether type 2 diabetes mellitus (T2DM) was a risk factor for the progression and development of CRC. A mouse model of a transplanted colorectal tumor with T2DM was established to observe the changes in volume and size of the transplanted tumor. Mice were sacrificed at the end of the 5th week to determine the serum IGF-1 level and VEGF expression in the tumor tissues. The tumor volume $\left(1628.5 \pm 882 \mathrm{~mm}^{3}\right)$ in the CRC-DM group was larger than that in the CRC group $\left(1950.2 \pm 726 \mathrm{~mm}^{3}\right.$; $\mathrm{P}<0.05)$. The serum IGF-1 level $(105.33 \pm 32.32 \mathrm{ng} / \mathrm{ml})$ was higher than that in the normal $(69.83 \pm 25.57 \mathrm{ng} / \mathrm{ml})$ and CRC groups $(70.17 \pm 25.27 \mathrm{ng} / \mathrm{ml} ; \mathrm{P}<0.05)$. The VEGF expression in the tumor tissues of the CRC-DM group $(70.0 \pm 11.5 \%)$ was higher than that in the $\mathrm{CRC}$ group $(42.9 \pm 7.5 \%$; $\mathrm{P}<0.05)$. T2DM may be one of the causes for the promotion of CRC growth and its mechanism may be correlated with the increased IGF-1 action observed in the blood that induces VEGF gene transcription, upregulates VEGF expression, causes tumor angiogenesis and thus leads to the occurrence and metastasis of tumors.
\end{abstract}

Correspondence to: Professor Defang Huang, Department of Endocrinology, The Third Affiliated Hospital of Nantong University, No. 585 Xingyuan Bei road, Wuxi, Jiangsu 214041, P.R. China E-mail: defanghuang@yeah.net

${ }^{*}$ Contributed equally

Key words: diabetes mellitus, type 2, colorectal cancer, VEGF, IGF-1, mice

\section{Introduction}

Colorectal cancer (CRC) is the joint name for colon and rectal cancer, one of the most common malignant tumors. Numerous scholars have observed that the occurrence and development of CRC is closely correlated with diabetes mellitus (DM) (1-4). The studies observed that the mechanism of DM-induced CRC may include the following: i) hyperglycemia, ii) the role of hyperinsulinemia and insulin resistance, iii) the role of insulin-like growth factor-1 (IGF-1), iv) the role of metabolic syndrome-related inflammatory factors (including adipokines) (5) for cell proliferation, v) the role of the immune system and vi) the increase of plasma endothelin level in diabetics, as well as the abnormalities of various trace elements, including chrome, zinc, manganese, selenium, magnesium and ferrum. The incidence of colorectal tumors caused by the increased insulin secretion from certain hypoglycemic agents may also be involved. In the present study, in order to further demonstrate the correlation between these factors, a type $2 \mathrm{DM}$ (T2DM) model was established based on a mouse model of a transplanted colorectal tumor. The changes in the mouse serum IGF-1 levels and the vascular endothelial growth factor (VEGF) expression were observed in the tumor in order to discuss whether type 2 diabetes was a significant risk factor for promoting the development and progression of CRC and to explain the possible mechanism behind this. The present study was conducted in the hope of providing a research basis for the prevention of CRC.

\section{Materials and methods}

Experimental groups. ICR mice were purchased from the Shanghai SLAC Laboratory Animal Center (Shanghai, China). The mice were 4-6 weeks old, weighed $22 \pm 2.0 \mathrm{~g}$ and were half males and half females. The mice were fed in a clean animal room [Certification Number SCXK (Shanghai) 2008-0005], at a room temperature of $22-25^{\circ} \mathrm{C}$. The bedding, drinking water and food were all sterilized. The mice were randomly divided into four groups; 8 mice comprising group A were the blank control group, 8 mice in group B were the DM group, 12 mice 
in group $\mathrm{C}$ were the CRC group and 12 mice in group $\mathrm{D}$ were the CRC-DM group. Groups B and D were provided a high-fat and high-sugar diet for four weeks and the other groups were provided a normal diet for four weeks. The present study was carried out in strict accordance with the recommendations in the Guide for the Care and Use of Laboratory Animals of the National Institutes of Health. The animal use protocol was reviewed and approved by the Institutional Animal Care and Use Committee (IACUC) of the Third Affiliated Hospital of Nantong University, Wuxi, Jiangsu, China.

Animal model. CT26 cells were suspended in $20 \%$ new-born calf serum and cell culture medium (RPMl-1640 nutrient solution containing $100 \mathrm{IU} / \mathrm{ml}$ penicillin and $100 \mu \mathrm{g} / \mathrm{ml}$ streptomycin), then cultivated with $5 \% \mathrm{CO}_{2}$ at $37^{\circ} \mathrm{C}$. The cells with adherent growth were removed following mechanical blowing and prepared to the desired cell suspension concentration with an appropriate amount of physiological saline. A $0.2 \mathrm{ml}$ suspension of $2 \times 10^{6}$ cancer cells was subcutaneously inoculated into the right axillary of the ICR mice. The cells were passaged twice in the mice and the tumor body was peeled off in $1 \mathrm{~mm}^{3}$ sections. These cells were then subcutaneously inoculated with a surgical trocar into the right axillary of the mice in groups $\mathrm{C}$ and $\mathrm{D}$. After 7 days, the mice with a tumor diameter of $\sim 0.5 \mathrm{~cm}$ were selected to establish the transplanted tumor model. Following the successful transplant of the tumors into groups $\mathrm{C}$ and $\mathrm{D}$, the mice in groups $\mathrm{B}$ and $\mathrm{D}$ were administered an intraperitoneal injection of $100 \mathrm{mg} / \mathrm{kg}$ streptozotocin (STZ) sodium citrate buffer (Sigma, St. Louis, MO, USA) and the normal control group was administered an intraperitoneal injection of $\mathrm{pH} 4.4$ sodium citrate buffer. A T2DM model was established when the blood sugar measured $>11.0 \mathrm{mmol} / 1$ following two consecutive tail amputations. Groups B and D continued to be provided with high-fat and high-sugar diets and the other groups were provided with normal diets.

Experiment detection. On the 3rd day subsequent to the establishment of the transferred tumor model, the long and short diameters of the subcutaneous tumor were measured with a vernier caliper to calculate the tumor volume and draw the tumor growth curves. The animals were sacrificed following 28 days of tumor growth and the subcutaneous tumor was peeled off and weighed with an electronic scale. The serum IGF-1 level was measured using a kit (Boster Biological Engineering Company, Wuhan, China), following the manufacturer's instructions. The values in each well were read at the $450 \mathrm{~nm}$ wavelength following all the surgical procedures. The standard curve was drawn on logarithmic coordinate paper according to the measured A-values of each standard substance and the test results of the serum specimens in all the laboratory mice were compared with the standard curve to obtain their serum IGF-1 levels. The VEGF expression in the tumor tissues was detected by immunohistochemistry (SP method). Claybank particles that appeared in the normal mucosa epithelial cells or in the cytoplasm of tumor cells were considered as evidence of positive expression. Positive expression was judged according to the standard of Volm: i) The uniformly colored tumor area was selected and the colored cells were counted using x400 magnification. The percentage of colored cells was calculated and represented the total number of cells in the respective field of vision. The means were taken and graded from 0-3 according to the percentage of the colored cells accounting for the total number of cells in the field of vision. Grade 0,0\%; grade 1, <25\%; grade 2, 25-56\%; and grade $3,>56 \%$. ii) The cells were graded according to the degree of coloration: Grade 0 , no color; grade 1, weakly colored; grade 2, moderately colored; and grade 3, strongly colored. The results were judged according to the sum of the i) and ii) values: negative, 0 ; weakly positive, $1-2$; moderately positive, 3-4; and strongly positive, 5-6.

Statistical analysis. The measurement data were presented as the mean $\pm \mathrm{SD}$ and all the indicator analyses were performed by SPSS 13.0 software. The inter-group comparison used LSD (least significant difference; homogeneity of variance) or Tamhane (heterogeneity of variance) tests for statistical analysis. $\mathrm{P}<0.05$ was considered to indicate a statistically significant difference.

\section{Results}

General observation. The mice in groups B and D developed a significant increase in weight subsequent to being fed with a high-fat and high-sugar diet for 4 weeks. The mice in groups $\mathrm{C}$ and D developed tumors, mostly elliptical in shape, following the inoculation with CRC cells and tumors were left to grow for 1 week, and two weeks later, an irregular and uneven surface gradually appeared. The tumors of the two groups continued to grow, but were more evident in the CRC-DM group. There were no significant differences in diet or activity between each group. No deaths occurred as a result of the experiment, with the exception of two mice that escaped from the blank control group.

Comparison of the change in tumor volume and weight between the CRC and CRC-DM groups. On the 7th day subsequent to the inoculation of the tumor cells in the CRC and the CRC-DM groups, the tumor tissues had grown and the tumor volumes had increased, although this was more evident in the CRC-DM group (Fig. 4). Subsequent to the experiment, the average weight of the transplanted tumors in the CRC-DM group was $2.30 \pm 0.43 \mathrm{~g}$, which was higher than that in the CRC group $(1.19 \pm 0.25 \mathrm{~g})$, with a statistically significant difference between the two groups. This indicated that the tumors based on type 2 diabetes were able to grow larger and more rapidly (Fig. 1 and Table I).

Comparison of serum IGF-1 values in each group. Compared with the control group, the serum IGF-1 levels in the CRC group were slightly higher, but with no statistically significant difference, while the serum IGF-1 levels in the DM and CRC-DM groups were increased with a statistically significant difference $(\mathrm{P}<0.05)$. Compared with the $\mathrm{CRC}$ group, the serum IGF-1 levels in the DM-CRC group were increased, with a statistically significant difference between the two groups $(\mathrm{P}<0.05)$. This indicated that the serum IGF-1 levels in the DM group were higher than those in the non-DM groups and that there were no significant changes in the serum IGF-1 levels subsequent to the transplantation of tumor cells when based on DM (Table II). 
Table I. Comparison of transplanted tumor weight in mice between the two groups.

\begin{tabular}{lcc}
\hline Groups & Number of mice & Tumor weight $(\mathrm{g})$ \\
\hline CRC & 12 & $1.19 \pm 0.25$ \\
DM-CRC & 12 & $2.30 \pm 0.43^{\mathrm{a}}$ \\
\hline
\end{tabular}

${ }^{\mathrm{a} C o m p a r e d}$ with the colorectal cancer group, $\mathrm{P}<0.05$. CRC, colorectal cancer; DM, diabetes mellitus.

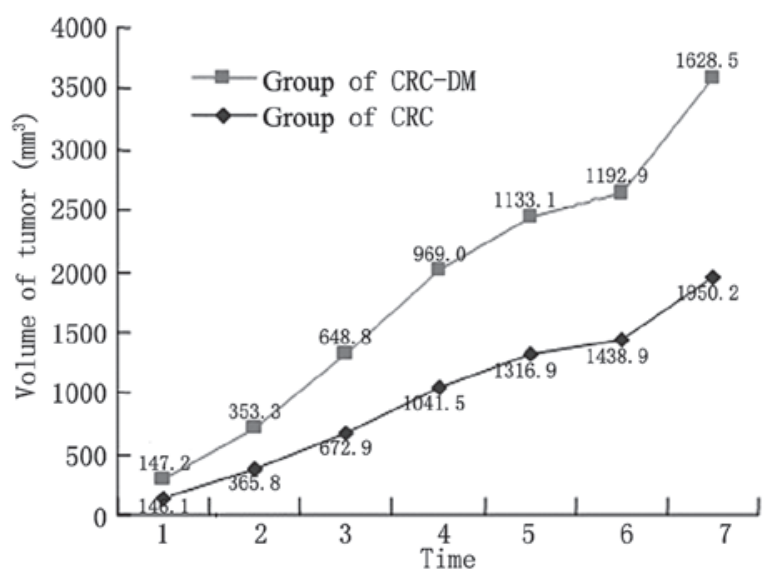

Figure 1. Tumor growth curves. CRC, colorectal cancer; DM, diabetes mellitus.

Table II. Serum levels of IGF-1.

\begin{tabular}{lcc}
\hline Groups & Number of mice & IGF-1 value (ng/ml) \\
\hline Blank control & 6 & $69.83 \pm 25.57$ \\
CRC & 12 & $70.17 \pm 25.27$ \\
DM & 8 & $103.63 \pm 31.67^{\mathrm{a}}$ \\
DM-CRC & 12 & $105.33 \pm 32.32^{\mathrm{a}, \mathrm{b}}$ \\
\hline
\end{tabular}

${ }^{\mathrm{a} C o m p a r e d ~ w i t h ~ t h e ~ c o n t r o l ~ g r o u p, ~} \mathrm{P}<0.05$; ${ }^{\mathrm{b}}$ Compared with the colorectal cancer group, $\mathrm{P}<0.05$. IGF-1, insulin-like growth factor- 1 ; CRC, colorectal cancer; DM, diabetes mellitus.

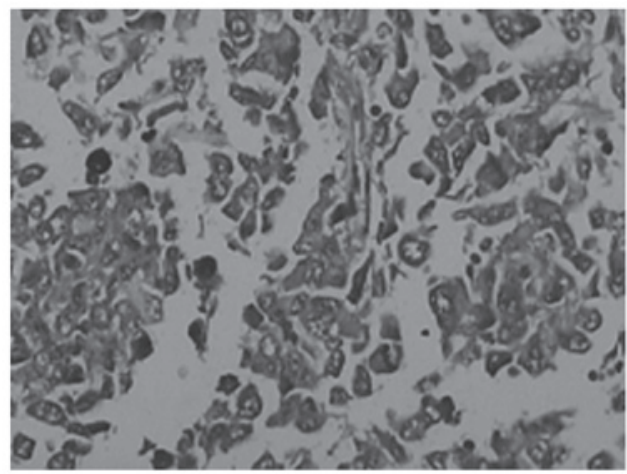

Figure 2. VEGF expression in a transplanted tumor of the CRC group. VEGF, vascular endothelial growth factor; $\mathrm{CRC}$, colorectal cancer.
Table III. Comparison of VEGF expression in the transplanted mouse tumors.

\begin{tabular}{lcc}
\hline Groups & Number of mice & VEGF expression (\%) \\
\hline CRC & 12 & $42.9 \pm 7.5$ \\
DM-CRC & 12 & $70.0 \pm 11.5^{\mathrm{a}}$ \\
\hline
\end{tabular}

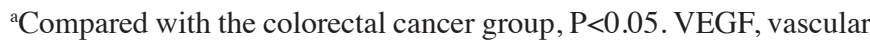
endothelial growth factor; CRC, colorectal cancer; DM, diabetes mellitus.

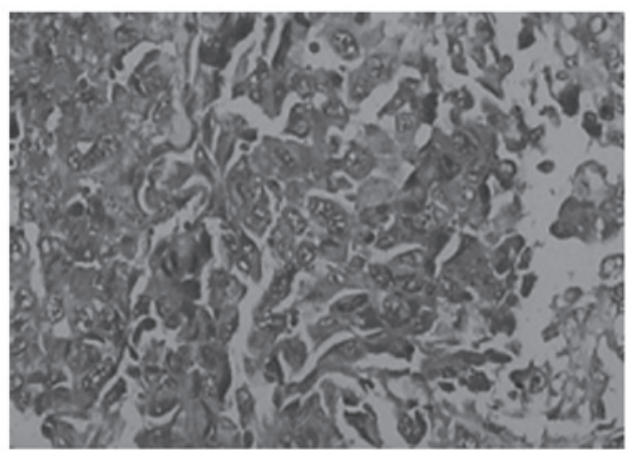

Figure 3. VEGF expression in a transplanted tumor in the CRC-DM group. VEGF, vascular endothelial growth factor; CRC, colorectal cancer.

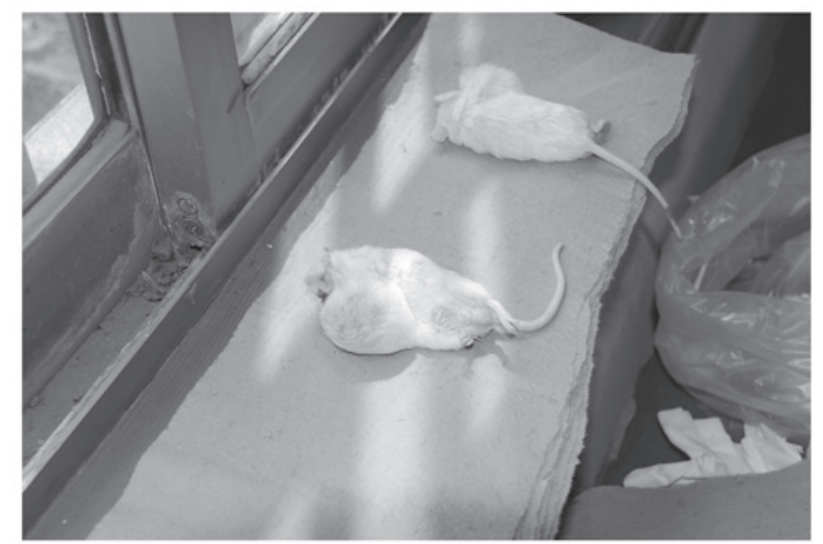

Figure 4. Mice in the colorectal cancer with diabetes (CRC-DM) group.

Comparison of VEGF expression in the tumor tissues between the CRC and CRC-DM groups. As shown in Table III, VEGF was primarily expressed in the cytoplasm of the tumor cells. There were varying levels of expression in the two groups, but a more evident expression was present in the CRC-DM group than in the CRC group ( $\mathrm{P}<0.05$; Table III, Figs. 2 and 3).

\section{Discussion}

In previous studies, the T2DM mouse models were established by the transplantation of tumor cells into normal and nude mice or by inducing insulin resistance with a high-fat 
diet (6), however, there were few studies on the concurrent establishment of a T2DM and colorectal transplanted tumor model. In the present study, the results of the concurrent establishment of the two models was shown. The colorectal transplanted tumors were passaged for only two generations, in line with the requirement of $<15$ generations for passaging in vivo, effectively preventing the repeated passages in the tumor body from causing a reduction in dissimilar types of cell phenotypes and changes in biological characteristics. Inoculation and passaging should be strictly performed under aseptic conditions. In the CRC-DM group, the tumor cells were transplanted after the mice were fattened. Subsequent to the growth of the tumor cells into a group of a certain size, STZ was injected again and the blood sugar level was measured until it was $>11.0 \mathrm{mmol} / \mathrm{l}$. At the same time, the transplanted tumor continued to grow; the tumor exhibited rapid growth during the overall study, with no growth failures or extinction phenomena, indicating that the mouse colorectal transplanted tumor had not been excluded for histoincompatibility, therefore providing a tumor specimen for the study.

IGF-1, also known as somatomedin C, is mainly synthesized and secreted by the liver. In the blood, IGF-1 and IGF-binding protein (IGFBP) exist in a complex form, but have no activity, and only combined with its specific receptors, IGF-1 was able to cause a series of biochemical reactions. Subsequent to IGF-1 combining with its receptors, the receptor is phosphorylated itself and causes various types of signal activation reactions, including those of the tyrosine kinase and phosphatidylinositol (IP)-3 kinase/protein kinase B (PKB) pathways. This may also activate the insulin receptor substrate (IRS) family proteins and cause certain biological effects using these pathways. It has been observed in previous studies that in the majority of patients with diabetes, the IGF-1 level was higher than in healthy individuals, while the IGFBP was lower (7). However, there have also been other studies that observed the blood IGF-1 and IGFBP levels in patients with T2DM and identified that the IGF-1 levels were increased only following the development complications. The present study observed that the mouse serum IGF-1 levels in the T2DM model were increased compared with the control group, which proved our previous assumptions that the IGF-1 level in T2DM patients was higher than in healthy individuals. Considering that the plasma insulin levels in T2DM show a delayed release with sugar stimulation, a previous study showed that the plasma insulin levels with hollow and sugar stimulation were often higher than the normal levels and that the plasma insulin levels were often able to show mitogenic characteristics and improve the IGF-1 level to achieve biological characteristics (7). Since IGFBP may be combined with IGF-1 to inhibit the physiological role of IGF-1, insulin may reduce the secretion of hepatogenic IGFBP and indirectly cause the increase in the IGF-1 level (8). IGF-1 may promote growth, development and metabolism, as well as the immunomodulatory effect. It has been demonstrated that IGF-1 is closely correlated with the growth and transfer of tumors. IGF-1 was observed with strong mitosis immunogenicity and anti-apoptotic activity and was able to be produced by the autocrine and paracrine function of tumor cells to promote the differentiation and growth of the cells (9). Exogenous IGF-1 as a growth factor may also promote the growth of the tumor cells. IGF-1 combines with its receptors to activate the PI3K/Akt and MAPK signaling pathways (10), inhibiting tumor cell apoptosis and promoting cell proliferation, respectively (11-14). In the present study, the results indicated that the growth of the transplanted colorectal tumor tissues from the mouse T2DM model was more rapid than that from the normal mice. The tumor volume and weight were also higher than in the normal mice, indicating that the high level of IGF-1 caused by T2DM was a significant factor for CRC occurrence and development.

VEGF, also termed vascular permeable factor, is a highly specific vascular endothelial cell mitogen. Its main biological functions include the ability to i) selectively enhance vascular endothelial cell mitosis to stimulate the endothelial cells proliferation and promote angiogenesis; and ii) strengthen the permeability of blood vessels, particularly small blood vessels, to conduct the extravasation and deposition of plasma proteins and other macromolecules into the extravascular matrix, providing nutrition for the establishment of a new capillary network of blood vessels. The biological activity of VEGF is mainly mediated by the tyrosine kinase receptor, with high affinity, and combines with its specific VEGF receptor (VEGFR) to cause a series of signal transductions, release a variety of cytokines and growth factors, stimulate vascular endothelial cell proliferation and migration and promote angiogenesis. This is important in the growth and metastasis of tumors (15). Studies $(16,17)$ have shown that VEGF expression is positively correlated with the infiltration depth, lymph node metastasis, distant metastasis and Dukes' staging of CRC, thereby affecting the growth and metastasis of tumors. The present study has shown that the growth of colorectal transplanted tumors from the mouse T2DM model was more evident than that from normal mice; the tumors volumes were larger and the VEGF expression of the tumor tissues was stronger, as observed by immunohistochemistry $(\mathrm{P}<0.05)$. Diabetes causes an increase in blood sugars and free fatty acids, providing energy for tumor growth. Long-term hyperglycemia would also cause the basement membrane of the capillary blood vessels to thicken and the permeability of the capillary blood vessels to decline. This would cause the enzyme system participating in the aerobic metabolism of cells to be damaged and also cause disorder in the aerobic metabolism process, with enhanced anaerobic glycolysis. It has been shown that the ability for glycolysis in normal cells is weaker than in tumor cells, therefore, the hyperglycemia state is more conducive to the growth of the tumor cells (18). This may be a reason for the more rapid growth of tumors based on diabetes. The present study has also indicated that the serum IGF-1 levels in the DM-CRC group were higher than those in the CRC group, while at the same time, the VEGF-positive expression rate in the tumor tissues was also stronger than that in the CRC group, indicating that they had a certain synergy to CRC growth. IGF-1 is able to stimulate endothelial cell migration and morphological differentiation to induce angiogenesis and may also be combined with the subendothelial extracellular matrix across vascular endothelial cells using the paracellular pathway. This may therefore play a role in endothelial cell subsistence and stabilization (19) and enhance the VEGF expression in the CRC cells, potentially resulting in the promotion of tumor angiogenesis and tumor growth and transfer, indicating that diabetes may promote tumor growth and that there is a link between them. 
However, the present study was only aimed to actively utilize tumor cells based on the mouse T2DM model in order to observe tumor growth. The study confirmed that diabetes was able to promote tumor growth, but did not prove that diabetes was able to induce tumor generation. VEGF was one of the cell signaling molecules used to promote the growth of the tumors. Additionally, the mechanism of T2DM in the induction of intestinal tumor generation was investigated to discuss the numerous types of cell signaling pathways that cause tumor growth.

\section{References}

1. Larsson SC, Orsini N and Wolk A: Diabetes mellitus and risk of colorectal cancer: a meta-analysis. J Natl Cancer Inst 97: $1679-1687,2005$

2. Meyerhardt JA, Catalano PJ, Haller DG, Mayer RJ, Macdonald JS, Benson AB III and Fuchs CS: Impact of diabetes mellitus on outcomes in patients with colon cancer. J Clin Oncol 21: 433-440, 2003.

3. Levi F, Pasche C, Lucchini F and La Vecchia C: Diabetes mellitus, family history, and colorectal cancer. J Epidemiol Community Health 56: 479-480, 2002.

4. Giouleme O, Diamantidis MD and Katsaros MG: Is diabetes a causal agent for colorectal cancer? Pathophysiological and molecular mechanisms. World J Gastroenterol 17: 444-448, 2011.

5. Pechlivanis S, Bermejo JL, Pardini B, et al: Genetic variation in adipokine genes and risk of colorectal cancer. Eur J Endocrinol 160: 933-940, 2009.

6. Kobayashi M, Ohno T, Tsuchiya T and Horio F: Characterization of diabetes-related traits in MSM and JF1 mice on high-fat diet. J Nutr Biochem 15: 614-621, 2004.

7. Ezzat VA, Duncan ER, Wheatcroft SB and Kearney MT: The role of IGF-I and its binding proteins in the development of type 2 diabetes and cardiovascular disease. Diabetes Obes Metab 10: $198-211,2008$

8. Ooi GT, Tseng LY, Tran MQ and Rechler MM: Insulin rapidly decreases insulin-like growth factor-binding protein-1 gene transcription in streptozotocin-diabetic rats. Mol Endocrinol 6 : 2219-2228, 1992.
9. Guastamacchia E, Resta F, Triggiani V, et al: Evidence for a putative relationship between type 2 diabetes and neoplasia with particular reference to breast cancer: role of hormones, growth factors and specific receptors. Curr Drug Targets Immune Endocr Metabol Disord 4: 59-66, 2004.

10. Lee CT, Park KH, Adachi Y, et al: Recombinant adenoviruses expressing dominant negative insulin-like growth factor-I receptor demonstrate antitumor effects on lung cancer. Cancer Gene Ther 10: 57-63, 2003.

11. Bai HZ, Pollman MJ, Inishi Y and Gibbons GH: Regulation of vascular smooth muscle cell apoptosis: Modulation of bad by a phosphatidylinositol 3-kinase-dependent pathway. Circ Res 85: 229-237, 1999.

12. Matsui T, Li L, del Monte F, et al: Adenoviral gene transfer of activated phosphatidylinositol 3'-kinase and Akt inhibits apoptosis of hypoxic cardiomyocytes in vitro. Circulation 100: 2373-2379, 1999 .

13. Ibrahim YH and Yee D: Insulin-like growth factor-I and cancer risk. Growth Horm IGF Res 14: 261-269, 2004

14. Kohda M, Hoshiya H, Katoh M, et al: Frequent loss of imprinting of IGF2 and MEST in lung adenocarcinoma. Mol Carcinog 31: 184-191, 2001.

15. Lin LJ, Zheng CQ, Jin Y, Ma Y, Jiang WG and Ma T: Expression of survivin protein in human colorectal carcinogenesis. World J Gastroenterol 9: 974-977, 2003

16. Minagawa N, Nakayama Y, Hirata K, et al: Correlation of plasma level and immunohistochemical expression of vascular endothelial growth factor in patients with advanced colorectal cancer. Anticancer Res 22: 2957-2963, 2002.

17. Hashim AF, Al-Janabi AA, Mahdi LH, Al-Toriahi KM and Yasseen AA: Vascular endothelial growth factor (VEGF) receptor expression correlates with histologic grade and stage of colorectal cancer. Libyan J Med 5: 2010.

18. Chang CK and Ulrich CM: Hyperinsulinaemia and hyperglycaemia: possible risk factors of colorectal cancer among diabetic patients. Diabetologia 46: 595-607, 2003

19. Grulich-Henn J, Ritter J, Mesewinkel S, Heinrich U, Bettendorf M and Preissner KT: Transport of insulin-like growth factor-1 across endothelial cell monolayers and its binding to the subendothelial matrix. Exp Clin Endocrinol Diabetes 110: 67-73, 2002. 